\title{
NUMERICAL ANALYSIS OF LARGE DEFLECTIONS OF A FLAT TEXTILE STRUCTURE WITH VARIABLE BENDING RIGIDITY AND VERIFICATION OF RESULTS USING FEM SIMULATION
}

\author{
Piotr Szablewski* \\ Department of Mechanical Engineering, Informatics and Chemistry of Polymer Materials, Lodz University of Technology, Lodz, Poland \\ ${ }^{*}$ Corresponding author. E-mail: piotr.szablewski@p.lodz.pl
}

\begin{abstract}
:
The paper presents the numerical modeling of large deflections of a flat textile structure subjected to a constant force acting on the free end. It was assumed that the examined structure is inextensible. The effect of the structure's own weight was also taken into account. In order to solve the problem, the flat textile structure was modeled using the heavy elastica theory. An important element of the analysis involves taking into account the variable bending rigidity of the examined textile structure along its length, which is often found in this type of products. The function of variable bending rigidity was assumed in advance. Numerical calculations were carried out in the Mathematica environment using the shooting method for the boundary value problem. The obtained results were verified using the finite element method.
\end{abstract}

\section{Keywords:}

Textile structure, large deflection, heavy elastica, variable bending rigidity, numerical methods, finite element method

\section{Introduction}

Deflection of cantilever beams has been the subject of numerous analyses to date. A good example of a cantilever beam subjected to a vertical concentrated force at the free end can be found in the book Mechanics of materials [1], as well as in many other textbooks on physics and mechanics. However, in the aforementioned textbook, the discussion only addresses beams subjected to small deflections. When deflections are large and the small-angle assumption is no longer valid, the problem becomes increasingly difficult, and an analytical solution does not exist due to the presence of a nonlinear term in the deflection equation. For the case of large deflection, several different solutions have been found for cantilever beams subjected to external forces.

Lee et al. [2] investigated large deflection of a linear elastic cantilever beam of variable cross section under combined loading by means of the Runge-Kutta-Falsi method. Baker [3] obtained large deflection profiles of linear elastic tapered cantilever beams under arbitrary distributed loads by means of a weighted residual solution of the Bernoulli-Euler bending moment equation. Dado and AL-Sadder [4] presented a new technique for the large deflection analysis of nonprismatic cantilever beams based on the integrated least-square error of the nonlinear governing differential equation in which the angle of rotation is represented by a polynomial. Shatnawi and AL-Sadder [5] studied the exact large deflection of nonprismatic, nonlinear bimodulus cantilever beams subjected to a tip moment by applying a power series approach to analytically solve highly nonlinear simultaneous first-order differential equations. Shvartsman [6] examined large deflections of a cantilever beam subjected to a follower force by reducing a nonlinear two-point boundary-value problem to an initial-value problem by the change of variables and then solving without iterations. Szablewski and Kobza [7] developed the numerical analysis of a test for the bending rigidity of textiles as proposed by Peirce. The mathematical model treated the textile product as an elastica that is subjected to large deflections. AL-Sadder and AL-Rawi [8] developed quasilinearization finite differences for the large deflection analysis of nonprismatic slender cantilever beams subjected to various types of continuous and discontinuous external variable distributed and concentrated loads in the horizontal and vertical global directions. Ibrahimbegovic [9] studied the large displacement of beams by implementing finite element (FE) analysis on the three-dimensional finite-strain Reissner beam theory, where the beam element reference axes are represented by arbitrary space-curved lines.

Cantilever beams of nonlinear materials have also been studied. Lewis and Monasa [10] numerically studied large deflections of cantilever beams made of nonlinear materials subjected to one vertical concentrated load at the free end using a fourth-order Runge-Kutta method. Lee [11] examined the large deflection of cantilever beams of a nonlinear elastic material under the effects of combined loading by using Butcher's fifth-order Runge-Kutta method. Szablewski and Korycki [12] analyzed 
the free folding of flat textiles by means of elastica for the same shape and folding conditions across the product. The problem was solved using the shooting method.

Rezazadeh [13] developed a comprehensive model to study the nonlinear behavior of multilayered micro beam switches for the application of microelectromechanical mechanical systems, in which the derived nonlinear equation was numerically solved using the nonlinear finite difference method. Antman [14] studied the large lateral buckling of nonlinearly elastic beams subjected to flexure, torsion, extension, or shear. This configuration is described by a position vector function and an orthonormal pair of vector functions of a real variable, which is interpreted as a scaled arc length parameter of the straight line of centroids of a beam in its natural reference configuration. Cesnik et al. [15] presented a refined theory of composite beams. The basis for the theory is the variational-asymptotical method, a mathematical technique by which the three-dimensional analysis of composite beam deformation can be split into a linear, two-dimensional cross-sectional analysis and a nonlinear, one-dimensional beam analysis. An original method of examining the state of equilibrium of a flat textile structure was presented by Szablewski [16]. This kind of structure was modeled as an inextensible elastica loaded with its own weight and axial force.

Large deflections of cantilever beams that are prismatic and made of linear elastic material have been the subject of numerous studies in which the beam is subjected to a uniformly distributed load. Seames and Conway [17] presented a numerical method for calculating large deflections of cantilever beams under uniform loading. This numerical method assumed that the elastic axis of the beam could be approximated by a number of circular arcs tangential to one another at their points of intersection, using the Bernoulli-Euler equation to determine the radius of each circular arc. Rhode [18] obtained an approximate solution for the large deflection of a cantilever beam subjected to a uniformly distributed load, by expanding the slope in a power series of the arc length. Lee et al. [19] analyzed stresses and displacements experimentally in largely deflected cantilever beams subjected to uniformly distributed loads by means of photoelasticity. This analysis demonstrated that for the case of a beam material having a small modulus of elasticity, with gravity acting alone as a uniform load, large deflections would occur.

Szablewski and Korycki [20] presented a general theory concerning the shape determination of coplanar elastica subjected to static bending by means of displacements. The displacements were described by the coordinates of the points of initially unbending elastica and the loads imposed. Belendez et al. [21] analyzed large deflections of a uniform cantilever beam under the action of a combined load consisting of a uniformly distributed load and an external vertical concentrated load applied at the free end. This analysis obtained a numerical solution using an algorithm based on the RungeKutta-Felhberg method and compared the numerical results with experimental results. Frisch-Fay [22] provided a solution for the large deflection of a cantilever beam subjected to two concentrated loads in terms of elliptic integrals. Bisshopp and
Drucker [23] provided a solution for the large deflection of a cantilever beam subjected to one concentrated load, acting vertically downward at the free end of the beam, also in terms of elliptic integrals. These two works $[22,23]$ are based on the fundamental Bernoulli-Euler theorem, which states that the curvature is proportional to the bending moment.

In this study, large deflection of textile structure with variable bending rigidity, treated as a beam subjected to a constant concentrated load applied at the free end, will be analyzed. Belendez et al. [24] attempted to find an exact analytical solution; however, upon discovering that one does not exist, they proceeded to apply a mixed numerical and analytical approach to provide solutions for the deflected shapes. This study will also attempt to find a numerical solution of the problem using Euler's numerical method along with the application of a shooting method using the Mathematica program to find the deflected shape. What sets this work apart from work performed by the aforementioned references is that this analysis will incorporate a constant, concentrated force applied to the free end at a constant angle and own weight of the tested object, thus not limiting the analysis to only a vertical downward concentrated force. However, due to the presence of a nonlinear term, an exact analytical solution does not exist. A numerical program using Euler's numerical method will be created to arrive at solutions for the shape of the deflected beam using the Mathematica environment, and a series of experiments will be performed to reproduce the program results. The obtained results will be verified using the FE method (FEM).

\section{Materials and methods}

\subsection{Numerical model of the examined object}

In this paper, during the run of the bending effect, the flat strip of the fabric will be represented as its longitudinal section. Here, the flat textile product is subjected to bending (e.g., woven fabric) of specified length $L$ and width $b$. The shape and bending conditions are the same across the product. Thus, the space three-dimensional problem can be reduced to an optional longitudinal cross section. Therefore, instead of studying the strip of fabric, the numerical analysis will be concerned with deflections of heavy elastica of a given bending rigidity $B$ and appropriate linear weight $q$, as shown in Figure 1.

Each point of the coordinate $s$, measured along the elastica, is defined by the Cartesian coordinates $x(s)$ and $y(s)$, where $x$ and $y$ are the functions of the variable $s$.

Furthermore, it will be assumed that the elastica is inextensible. It should also be pointed out that the assumption of inextensibility is some limiting. However, this assumption is often made in large-deflection analysis. In addition to the linear weight, the elastica is loaded with concentrated force. It will also be assumed that the cross section of the elastica remains constant across the length of the elastica, meaning that the effect of Poisson's ratio can be neglected. Next, it is assumed that the Bernoulli-Euler theorem is valid, which states that 


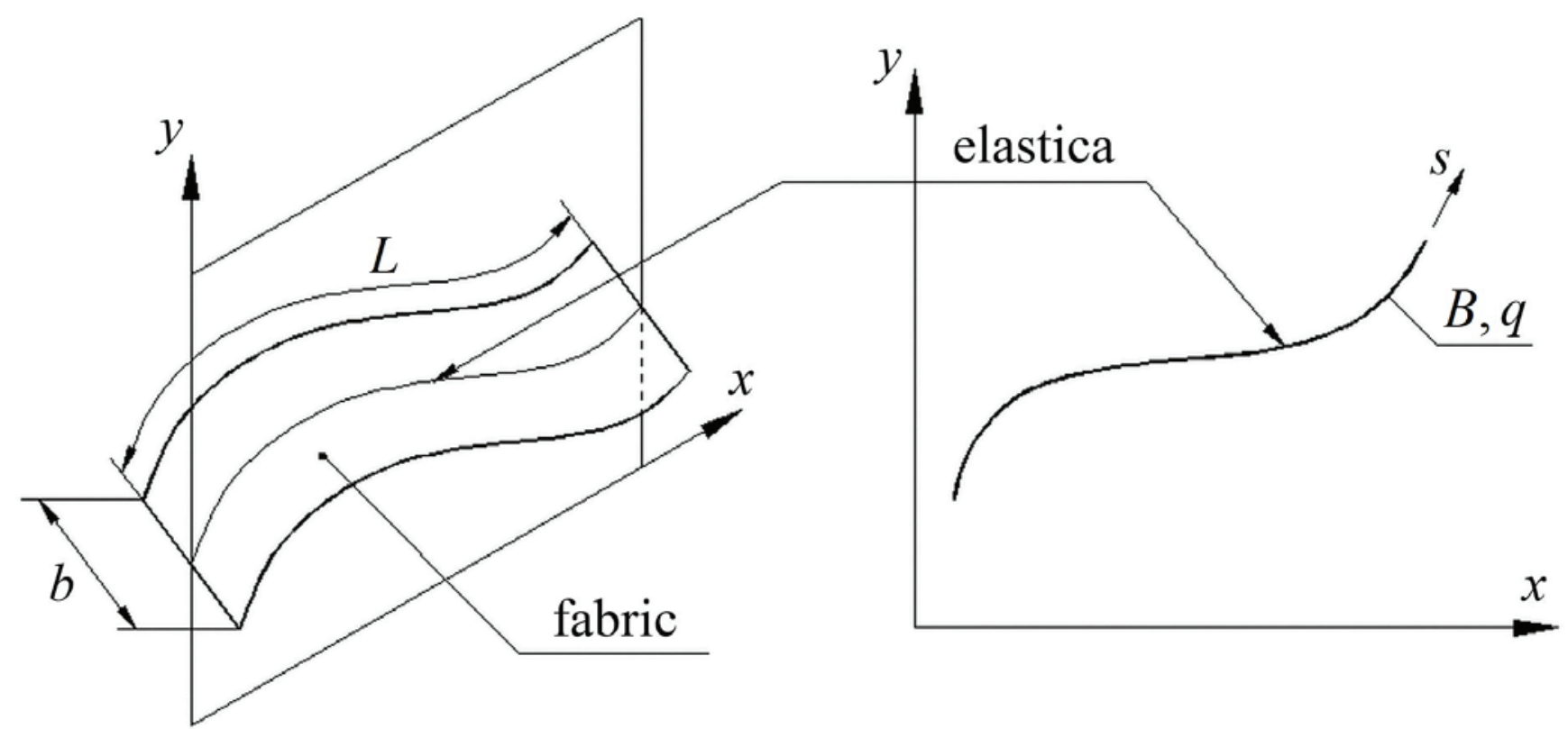

Figure 1. Model of the flat textile product approximated by elastica.

the curvature of the elastica is proportional to the bending moment. Figure 2 shows the examined object of length $L$ with a concentrated force $P$ applied at the free end. In this figure, $d x$ and $d y$ are the horizontal and vertical displacements at the free end, respectively, and $\varphi_{0}$ represents the maximum slope angle of the elastica.

The constant angle at which the force is applied is represented by $\alpha$, and it is measured positive downward from the horizontal axis. The coordinate $s$ shall be measured between the fixed end and point $A$.

A free body diagram, shown in Figure 3, describes the forces acting on the deflected object. In this study, $x(s)$ and $y(s)$ will be represented as $x$ and $y$. At the fixed end, labeled as $O$, $M_{\mathrm{O}}$ is the reaction moment, and $R_{\mathrm{x}}$ and $R_{\mathrm{y}}$ are the reaction forces. The force $P$ is resolved into the horizontal and vertical components $\left(P_{x}, P_{y}\right)$. It should be pointed out that the center of gravity coordinate $x$ of the deflected curve of length $L$ is

$$
x_{\mathrm{C}}=\left(\int_{0}^{L} x d s\right) \cdot L^{-1}
$$

The reaction moment $M_{\mathrm{O}}$ is expressed as follows:

$$
M_{\mathrm{O}}=P \sin \alpha(L-\delta x)+P \cos \alpha \delta y+q L \cdot\left(\int_{0}^{L} x d s\right) \cdot L^{-1}
$$

The equilibrium conditions yield the expression

$$
R_{x}=P \cos \alpha, R_{y}=P \sin \alpha+q L, M_{\mathrm{o}}=P \sin \alpha(L-\delta x)+P \cos \alpha \delta y+q \int_{0}^{L} x d s
$$

The Bernoulli-Euler bending moment-curvature equation for a uniform cross section of a rectanaular beam of linear elastic material is $E I \cdot(d \varphi / d s)=M(x, y)$, where $M(x, y)$ is the bending moment as a function of the distances $x$ and $y, \varphi$ represents the slope angle at any point along the length of the elastica, $E$ is the modulus of elasticity, and $I$ is the moment of

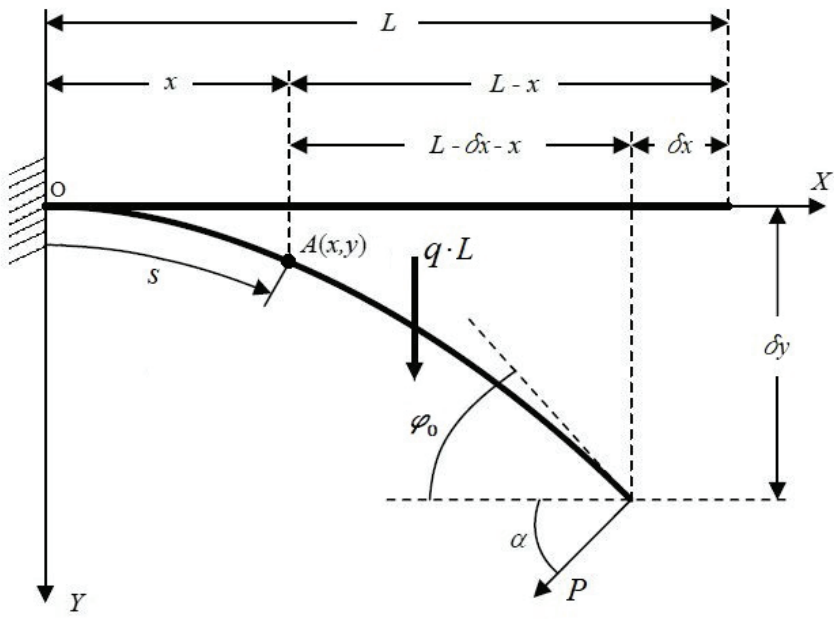

Figure 2. Examined object with imposed loads.

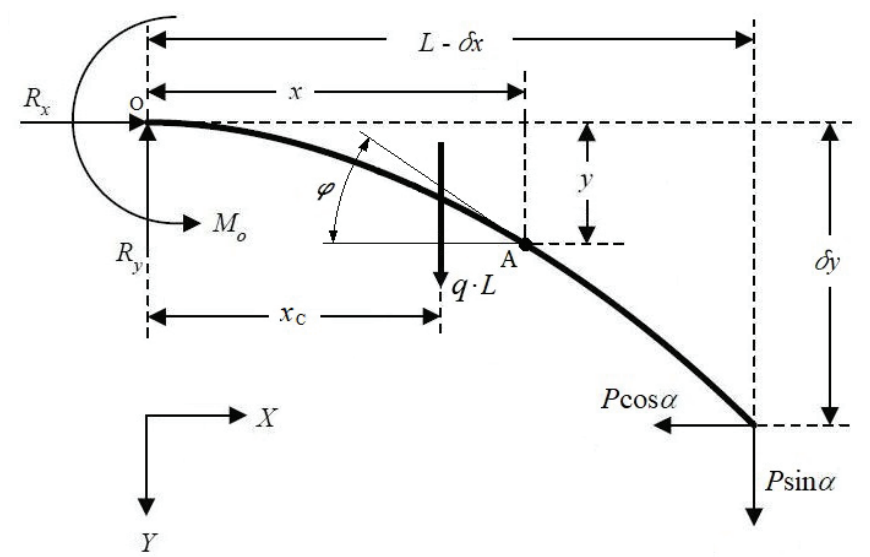

Figure 3. Free body diagram of the object.

inertia of the elastica cross section about the neutral axis. Let us denote the bending rigidity as $B$; therefore, $E I=B$ and

$$
B \frac{d \varphi}{d s}=M(x, y) \text {. }
$$


$B=B(s)$ is the variable bending rigidity as a function of the variable s. It is necessary to find $M$ as a function of $x$ and $y$ to obtain the moment at any point along the length of the elastica. This can be done by cutting the elastica at an arbitrary point and summing the moments about the cut point.

Figure 4 shows the cut elastica with the reactions and moments acting on it. The arc length of the elastica, which is measured between the fixed end $\mathrm{O}$ and point $\mathrm{A}$, is represented by $s$. At point $\mathrm{A}, M(x, y)$ is the moment $M$ as a function of the distances $x$ and $y$, while $V$ and $H$ represent the internal forces. The center of gravity coordinate $x$ of the deflected curve of length $s$ is $x_{c}=\left[\int_{0}^{s} x(\tau) d \tau\right] \cdot s^{-1}$. Summing the moments about A to obtain the moment $M$ as a function of $x$ and $y$ yields the expression

$$
M_{\mathrm{O}}-R_{x} y-R_{y} x+q s\left\{x-\left[\int_{0}^{s} x(\tau) d \tau\right] \cdot s^{-1}\right\}-M(x, y)=0
$$

Using Eqs (2) and (3), we have

$$
B \frac{d \varphi}{d s}=P \sin \alpha(L-\delta x-x)+P \cos \alpha(\delta y-y)-q L x+q \int_{0}^{L} x d s+q s x-q \int_{0}^{s} x(\tau) d \tau
$$

Taking the derivative of Eq. (5) with respect to $s$ and noting that $L, d x$, and $d y$ are constants, we get the following expression:

$$
\frac{d B}{d s} \frac{d \varphi}{d s}+B \frac{d^{2} \varphi}{d s^{2}}=-P \sin \alpha \frac{d x}{d s}-P \cos \alpha \frac{d y}{d s}-q L \frac{d x}{d s}+q\left[x+\frac{d x}{d s} s\right]-q x
$$

In Eq. (6), it was considered that if $F(s)=\int_{a}^{s} f(t) d t$ then $\frac{d F(s)}{d s}=f(s)$, (the basic theorem of calculus). Using trigonometry, the following relationships can be established:

$$
\frac{d x}{d s}=\cos \varphi, \quad \frac{d y}{d s}=\sin \varphi
$$

It is finally obtained that

$$
B \frac{d^{2} \varphi}{d s^{2}}=-P \sin \alpha \cos \varphi-P \cos \alpha \sin \varphi-q L \cos \varphi+q s \cos \varphi-\frac{d B}{d s} \frac{d \varphi}{d s}
$$

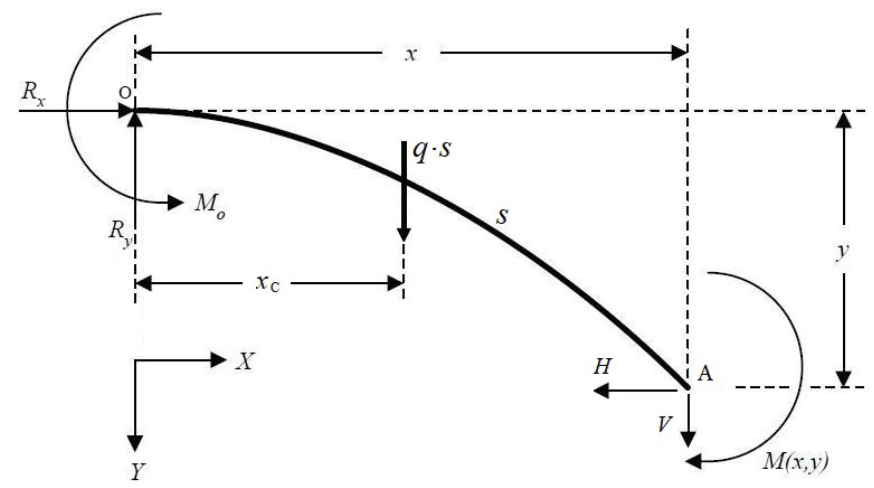

Figure 4. Free body diagram of the left portion of elastica up to the point $A$.
Eq. (8) is the nonlinear differential equation describing the deflection curve of a cantilever elastica made of linear elastic material subjected to a concentrated end load as shown in Figure 2. Because no accurate analytical solution exists, a numerical solution will be developed.

\subsection{Numerical solution of the problem}

Using Euler's method, the second-order nonlinear differential Eq. (8) can be reduced into two first-order nonlinear differential equations. The curvature of the elastica, denoted as $\mathrm{K}$, can be written as follows:

$$
\kappa=\frac{d \varphi}{d s}
$$

Substituting Eq. (9) into Eq. (8) yields

$B \frac{d \kappa}{d s}=-P \sin \alpha \cos \varphi-P \cos \alpha \sin \varphi-q L \cos \varphi+q s \cos \varphi-\frac{d B}{d s} \kappa$

and rearranging, we get

$\frac{d \kappa}{d s}=-\frac{P}{B} \sin \alpha \cos \varphi-\frac{P}{B} \cos \alpha \sin \varphi-\frac{q L}{B} \cos \varphi+\frac{q}{B} s \cos \varphi-\frac{d B(s) / d s}{B} \kappa$

To solve this problem numerically, a system of four ordinary first-order differential equations with four unknowns will be used. The system of equations is as shown below:

$$
\left\{\begin{array}{l}
\frac{d \varphi}{d s}=\kappa \\
\frac{d x}{d s}=\cos \varphi \\
\frac{d y}{d s}=\sin \varphi \\
\frac{d \kappa}{d s}=-\frac{P}{B} \sin \alpha \cos \varphi-\frac{P}{B} \cos \alpha \sin \varphi-\frac{q L}{B} \cos \varphi+\frac{q}{B} s \cos \varphi-\frac{d B / d s}{B} \kappa
\end{array}\right.
$$

Due to the fact that we are dealing with a typical boundary problem (not all initial values of the studied variables are known), the shooting method will be used to obtain the correct curvature at the fixed end. In order to solve the task, the Mathematica program was used with the appropriate function to solve the boundary problem using the shooting method. At the fixed end, $x, y$, and $\varphi=0$ are known. The curvature $\mathrm{K}$ is unknown. At the free end, the curvature $\mathrm{k}$ is known $(\mathrm{k}=0)$, and $x, y$, and $\varphi$ are unknown. The idea of the shooting method is to use the Newton-Raphson method to find such an initial value of curvature that at the free end, the curvature has a given value $\mathrm{K}=0$.

\section{Results and discussion}

\subsection{Modeling of variable bending rigidity}

The following data describing the examined object were adopted for numerical analysis. The length of the object $L=0.3 \mathrm{~m}$. The cross-sectional dimensions at the fixed and free points: width $b=0.02 \mathrm{~m}$, height $h_{0}=0.005 \mathrm{~m}$. The moment of inertia of a section: $I_{0}=\left(b h_{0}^{3}\right) / 12$. Young's modulus $E=2 \times 10^{8} \mathrm{~N} / \mathrm{m}^{2}$. Linear weight $q=1 \mathrm{~N} / \mathrm{m}$. An important element of the analysis 
is that the variable bending rigidity $B(s)$ of the examined textile structure along its length, which is often found in composite elements, is taken into account. The function of the variable bending rigidity was assumed in advance. The distribution of bending rigidity according to the second-degree polynomial was assumed. The function $B(s)$ is as follows:

$$
B(s)=\frac{B_{0}}{L^{2}}\left[L^{2}+4 L s(\xi-1)-4 s^{2}(\xi-1)\right]
$$

The function $B(s)$ is parabolic. According to Eq. (13), at the fixed and free points, the bending rigidity has a maximum value $B_{0}=E \cdot I_{0}$. At the midpoint of the elastica, the bending rigidity has a minimum value $\xi \cdot B_{0}$, where $\xi<1$. For the above data and $\xi=0.1$, we have $B_{0}=0.0416667 \mathrm{Nm}^{2}$ and

$$
B(s)=0.462963 \cdot\left(0.09-1.08 \cdot s+3.6 \cdot s^{2}\right)
$$

The graph of the function $B(s)$ is given below.

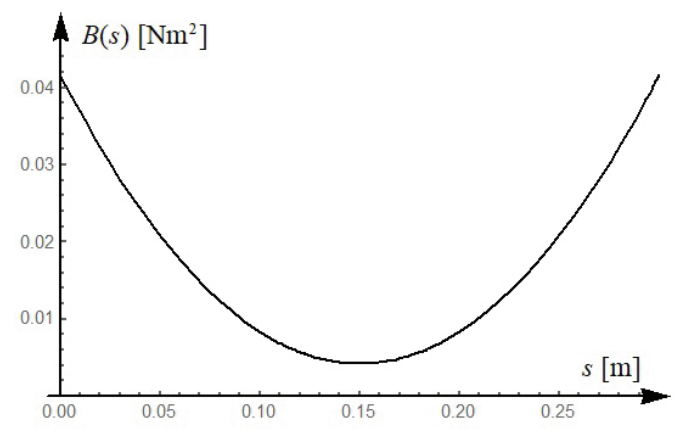

Figure 5. The graph of the function $B(s)$.

Referring to the bending length (according to Peirce's cantilever test), we have the following:

- $\quad$ maximum bending length for $B_{0}: D_{\max }=0.346681 \mathrm{~m}$;

- $\quad$ minimım bending length for $\xi \cdot B_{0}: D_{\min }=0.160915 \mathrm{~m}$, where $D=\sqrt[3]{\frac{B}{q}}$.

In order to verify the obtained results, an FEM model of the object was created using SolidWorks software. Variable bending rigidity was obtained through the variable section height $h(s)$, as shown below:

$h(s)=\sqrt[3]{\frac{12 B(s)}{E b}}=\sqrt[3]{\frac{12 B_{0}\left[L^{2}+4 L s(\xi-1)-4 s^{2}(\xi-1)\right]}{E b L^{2}}}$

The graph of the function $h(s)$ is given below.

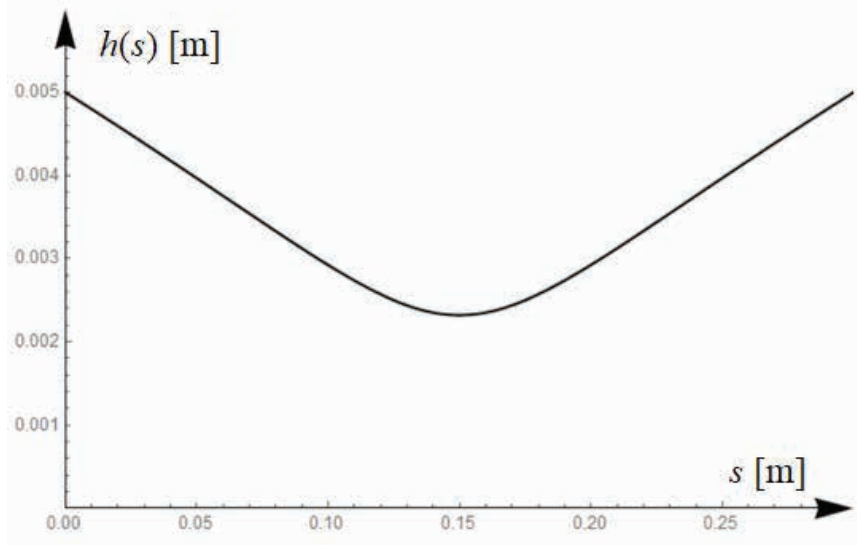

Figure 6. The graph of the variable section height $h(s)$.

Figure 7 shows the examined textile structure modeled in SolidWorks software.

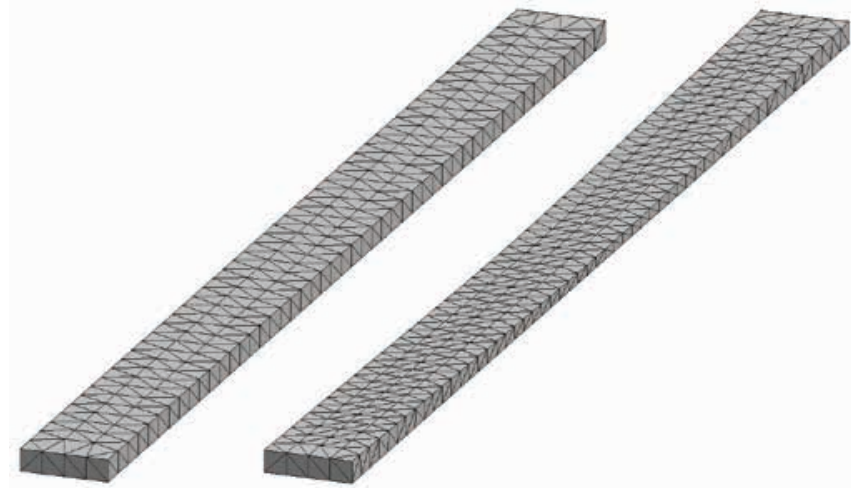

(a)

(b)

Figure 7. The examined textile structure modeled in SolidWorks software: a) constant bending rigidity; and b) variable bending rigidity.

\subsection{The results of calculations}

The calculations were carried out first for the load with own weight only $(P=0)$ and then together with the force acting on the free end of the structure $(P \neq 0)$.

The results of the calculation for the described analytical model are presented in Figure 8 . The value of the concentrated force $P$ applied at the free end is taken as $P=0.4 \mathrm{~N}$. The constant angle at which the force is applied is $\alpha=\pi / 2$.

The results for the FE model are presented in Figure 9. Calculations were carried out for the same data as for the analytical model. Solidworks software was used. To maintain the same loading conditions, the FE model must be subjected to a vertical pressure, which is $Q=q / b=50 \mathrm{Nm}^{-2}$.

Based on the analysis of the graphs (Figures 8 and 9), it can be stated that the shapes of the bent structure for both the analytical model and the FE model are identical. This is also confirmed by the analysis of the displacement values of the free 


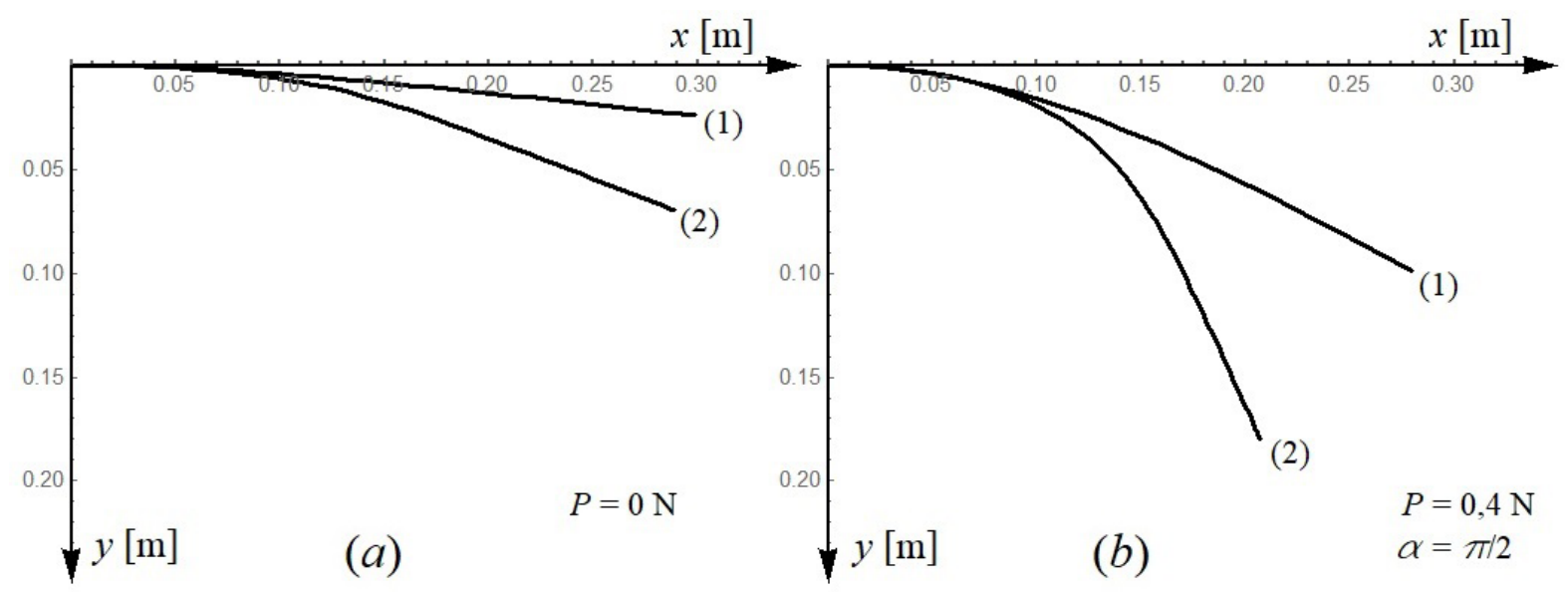

Figure 8. Results of the calculation according to the analytical model: (a) load with only own weight $(P=0 \mathrm{~N})$; $(\mathrm{b})$ own weight together with the force $(P=0.4 \mathrm{~N})$; (1) constant bending rigidity $B_{0}$; and (2) variable bending rigidity $B(s)$.

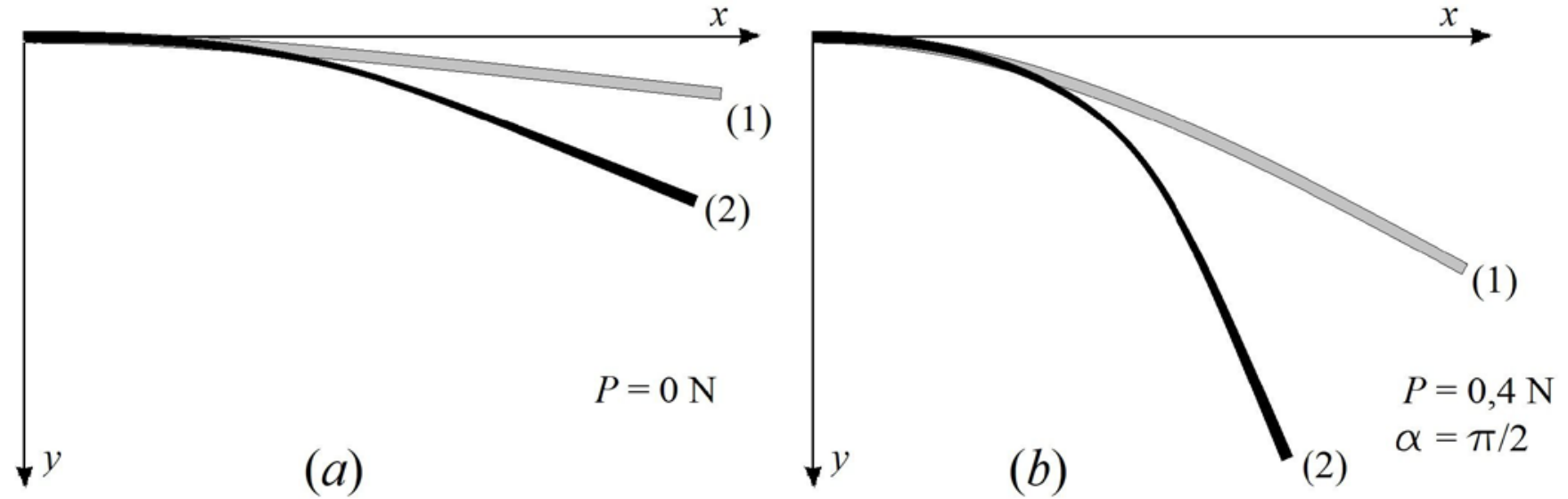

Figure 9. Results of calculation according to the FE model: (a) the load with own weight only $(P=0 \mathrm{~N})$; (b) own weight together with the force $(P=0.4 N)$; (1) constant bending rigidity $B 0$; and (2) variable bending rigidity $B(s)$.

Table 1. The horizontal and vertical displacement values for the analytical model and the FE model for selected loading conditions

\begin{tabular}{|c|c|c|c|c|c|}
\hline & & & Analytical model & $\begin{array}{c}\text { FE model } \\
\text { (SolidWorks) }\end{array}$ & $\begin{array}{l}\text { The relative error } \\
\text { of analytical model } \\
\text { compared to FE } \\
\text { analysis e, \% }\end{array}$ \\
\hline \multirow{4}{*}{$\begin{array}{c}\text { Constant } \\
\text { bending rigidity, } \\
B_{0}=\text { constant }\end{array}$} & \multirow{2}{*}{$P=0 \mathrm{~N}$} & $d x[\mathrm{~mm}]$ & 1.115 & 1.113 & 0.180 \\
\hline & & $d y[\mathrm{~mm}]$ & 24.174 & 24.148 & 0.108 \\
\hline & \multirow{2}{*}{$\begin{array}{c}P=0.4 \mathrm{~N} \text { for } \\
\alpha=\pi / 2\end{array}$} & $d x[\mathrm{~mm}]$ & 20.088 & 20.269 & 0.893 \\
\hline & & $d y[\mathrm{~mm}]$ & 98.906 & 99.286 & 0.383 \\
\hline \multirow{4}{*}{$\begin{array}{c}\text { Variable bending } \\
\text { rigidity, } B(s)\end{array}$} & \multirow{2}{*}{$P=0 \mathrm{~N}$} & $d x[\mathrm{~mm}]$ & 11.210 & 11.430 & 1.925 \\
\hline & & $d y[\mathrm{~mm}]$ & 69.778 & 69.910 & 0.189 \\
\hline & \multirow{2}{*}{$\begin{array}{c}P=0.4 \mathrm{~N} \text { for } \\
\alpha=\pi / 2\end{array}$} & $d x[\mathrm{~mm}]$ & 93.081 & 94.900 & 1.917 \\
\hline & & $d y[\mathrm{~mm}]$ & 180.205 & 180.100 & 0.058 \\
\hline
\end{tabular}


end for both models. The horizontal and vertical displacement values $d x$ and $d y$ are presented in Table 1 .

\section{Conclusion}

Analytical analysis of the large deflection of a cantilever textile structure subjected to a constant concentrated force with a constant angle applied at the free end and its own weight has been carried out. An attempt to find an exact analytic expression for both $x$ - and $y$-coordinates along the length of the deflected beam was made; however, the expressions for $d x$ and $d y$ could not be integrated. A numerical analysis was successfully performed, and a Mathematica program was written that would perform Euler's numerical method to find the $x$ - and $y$-coordinates along the length of the deflected structure for a given combination of beam geometry, material, and force. Then, the object deformation analysis was performed using the FE method based on SolidWorks software. FE analysis was used to verify the results obtained from the analytical model. Analysis of the bent shapes of the tested object presented in Figures 8 and 9 showed a high compatibility of the analytical model with the FE model. This is clearly seen by analyzing the results in Table 1. Finally, the correctness of the analytical model describing the presented bending case was found. The work will be the starting point for the so-called inverse analysis (synthesis), in which the bending rigidity of the object will be determined to obtain the given shape of the bent structure.

\section{References}

[1] Gere, J. M. (2004). Mechanics of materials. (6th ed.). Brooks/Cole-Thomson Learning (Belmont, CA).

[2] Lee, B. K., Wilson, J. F., Oh, S. J. (1993). Elastica of cantilevered beams with variable cross sections. International Journal of Non-Linear Mechanics, 28, 579589.

[3] Baker, G. (1993). On the large deflections of non-prismatic cantilevers with a finite depth. Computers \& Structures, 46(2), 365-370.

[4] Dado, M., AL-Sadder, S. (2005). A new technique for large deflection analysis of non-prismatic cantilever beams. Mechanics Research Communications, 32(6), 692-703.

[5] Shatnawi, A., AL-Sadder, S. (2007). Exact large deflection analysis of non-prismatic cantilever beams of nonlinear bimodulus material subjected to tip moment. Journal of Reinforced Plastics and Composites, 26(12), 1253-1268.

[6] Shvartsman, B. S. (2007). Large deflections of a cantilever beam subjected to a follower force. Journal of Sound and Vibration, 304(3-5), 969-973.

[7] Szablewski, P., Kobza, W. (2003). Numerical analysis of Peirce's cantilever test for the bending rigidity of textiles. Fibres \& Textiles in Eastern Europe, 11(4), 54-57.

[8] AL-Sadder, S., AL-Rawi, R. (2006). Finite difference scheme for large-deflection analysis of non-prismatic cantilever beams subjected to different types of continuous and discontinuous loadings. Archive of Applied Mechanics, 75(8), 459-473.
[9] Ibrahimbegovic, A. (1995). On finite element implementation of geometrically nonlinear Reissner's beam theory: Three-dimensional curved beam elements. Computer Methods in Applied Mechanics and Engineering, 122, 1126.

[10] Lewis, G., Monasa, F. (1981). Large deflections of cantilever beams of nonlinear materials. Computers \& Structures, 14(5-6), 357-360.

[11] Lee, K. (2002). Large deflections of cantilever beams of non-linear elastic material under a combined loading. International Journal of Non-Linear Mechanics, 37(3), 439443.

[12] Szablewski, P., Korycki, R. (2019).Numerical analysis of free folding of flat textile products and proposal of new test concerning bending rigidity. Indian Journal of Fibre \& Textile Research, 44, 180-187.

[13] Rezazadeh, G. (2008). A comprehensive model to study nonlinear behavior of multilayered micro beam switches. Microsystem Technologies, 14(1), 135-141.

[14] Antman, S. (1984). Large lateral buckling of nonlinearly elastic beams. Archive for Rational Mechanics and Analysis, 84(4), 293-305.

[15] Cesnik, C., Sutyrin, V., Hodges, D. (1996). Refined theory of composite beams: The role of short-wavelength extrapolation. International Journal of Solids and Structures, 33(10), 1387-1408.

[16] Szablewski, P. (2006). Analysis of the stability of a flat textile structure. AUTEX Research Journal, 6(4), 204-215.

[17] Seames, A. E., Conway, H. D. (1957). A numerical procedure for calculating the large deflections of straight and curved beams. Journal of Applied Mechanics, 24, 289294.

[18] Rhode, F. V. (1953). Large deflections of cantilever beams with uniformly distributed load. Quarterly of Applied Mathematics, 11, 337-338.

[19] Lee, H., Durelli A. J., Parks, V. J. (1969). Stress in largely deflected cantilever beams subjected to gravity. Journal of Applied Mechanics, 36, 323-325.

[20] Szablewski, P., Korycki, R. (2016). Shape determination of elastica subjected to bending by means of displacements. Fibres \& Textiles in Eastern Europe, 24(6), 138-142.

[21] Belendez, T., Perez-Polo, M., Neipp, C., Belendez, A. (2005). Numerical and experimental analysis of large deflections of cantilever beams under a combined load. Physica Scripta, T118, 61-65.

[22] Frisch-Fay, R. (1962). Large deflections of a cantilever beam under two concentrated loads. Journal of Applied Mechanics, 29(1), 200-201.

[23] Bisshopp, K. E., Drucker, D. C. (1945). Large deflections of cantilever beams. Quarterly of Applied Mathematics, 3, 272-275

[24] Belendez, T., Neipp, C., Belendez, A. (2002). Large and small deflections of a cantilever beam. European Journal of Physics, 23(3), 371-379. 